\title{
Effect of longitudinal magnetic and density inhomogeneity on transversal coronal loop oscillations
}

\author{
G. Verth and R. Erdélyi \\ Solar Physics and Space Plasma Research Centre (SP $\left.{ }^{2} \mathrm{RC}\right)$, University of Sheffield, Hicks Building, Hounsfield Road, Sheffield, \\ S3 7RH, UK \\ e-mail: [G.Verth;Robertus]@sheffield.ac.uk
}

Received 21 February 2008 / Accepted 28 May 2008

ABSTRACT

\begin{abstract}
Context. Observations of post-flare transversal coronal loop oscillations by TRACE have given us an excellent opportunity to implement magneto-seismological techniques for probing the plasma fine structure of the Sun's upper atmosphere.

Aims. We investigate the combined effect of magnetic and density stratification on transversal coronal loop oscillations.

Methods. A coronal loop will be modelled as an expanding magnetic flux tube with arbitrary longitudinal plasma density. The governing equation of the fast kink body mode is derived and solved by analytical approximation and numerical methods.

Results. It is found that even a relatively small coronal loop expansion can have a significant and pronounced effect on the accuracy of the plasma density scale height measurements derived from observation of loop oscillations.

Conclusions. To conduct more accurate and realistic magneto-seismology of coronal loops, the magnetic field divergence should be taken into account.
\end{abstract}

Key words. Sun: corona - Sun: magnetic fields - Sun: oscillations - Sun: fundamental parameters

\section{Introduction}

Solar post-flare transversal coronal loop oscillations observed by high-resolution EUV imager onboard the Transition Region And Coronal Explorer (TRACE) have provided valuable data to probe the plasma fine structure of the corona by implementing magneto-seismological techniques (see Banerjee et al. 2007, for the latest review). These magnetoacoustic oscillations have been observed many times, e.g., Aschwanden et al. (1999a), Aschwanden et al. (2002), Nakariakov et al. (1999) and Verwichte et al. (2004) and have been identified as the standing fast kink mode from MHD wave theory developed by e.g., Edwin \& Roberts (1983). This theory models a coronal loop as a straight magnetic cylinder with different external and internal plasma densities, both of which are taken to be constants. However, it is apparent from the wealth of many space-borne observations that the structure of coronal loops may be more complex than this simple equilibrium configuration.

Regarding plasma density in the solar atmosphere, it has been shown using spectroscopic methods that the density decreases drastically from the photosphere to the corona, e.g., Vernazza et al. (1981) estimated that for a particular active region the number density of electrons in the photosphere is $\approx 10^{14}-10^{15} \mathrm{~cm}^{-3}$ and only $\approx 10^{9} \mathrm{~cm}^{-3}$ in the lower corona. Attempts to measure the degree of plasma stratification in coronal loops using emission measure from EUV imager data have provided some puzzling results. In younger active region loops there have been measurements of "super-hydrostatic" scale heights that are up to four times higher than expected (Aschwanden et al. 2000, 2001). On the other hand, loops have been observed in older active regions that are close to hydrostatic equilibrium with density scale heights that can be explained by gravitational stratification (Aschwanden et al. 1999b). To further complicate matters the plasma of "static" coronal loops has also exhibited significant dynamic behaviour such as flows (Brekke et al. 2001; Winebarger et al. 2001, 2002) and cooling events (Schrijver 2001; Winebarger et al. 2003). It was shown by Terra-Homem et al. (2003) that flows can significantly alter the oscillation frequencies of coronal loops.

To investigate how plasma density stratification in coronal loops affects the standing fast kink body mode, more realistic MHD wave theory has been developed which can model a loop as a magnetic cylinder with an inhomogeneous plasma density equilibrium. Following on from the work of Hollweg \& Yang (1988), it has been shown that varying plasma density in the radial direction results in a change of period and damping of MHD waves (see e.g., Ruderman \& Roberts 2002; Goossens et al. 2002; Aschwanden et al. 2003; Van Doorsselaere et al. 2004; Arregui et al. 2007). Furthermore, inhomogeneity in the longitudinal direction of coronal loops leads to changes in the amplitude profiles of standing modes and the ratios of the periods of the fundamental mode to that of higher harmonics (see e.g., Andries et al. 2005a,b; Goossens et al. 2006; Dymova \& Ruderman 2006a,b; McEwan et al. 2006; Arregui et al. 2007; Erdélyi \& Verth 2007; Verth et al. 2007). Applying this theory to TRACE data of loop oscillations, attempts have been made to estimate coronal density scale heights (see e.g., Andries et al. 2005a; Van Doorsselaere et al. 2007). However, the theory used for this type of magneto-seismology assumed a constant magnetic field along loops. Since the kink speed in a homogeneous loop is also proportional to the magnetic field strength, magnetic inhomogeneities will also affect the properties of these oscillations as shown by Verth (2007) (referred to as V07 hereafter). It is very difficult to make direct measurements of magnetic field strength variation in the corona, although recently there has been some progress made using spectropolarimetry by 
Lin et al. (2004). On average, the magnetic field strength is expected to decrease with height above the photosphere and the results of Lin et al. (2004) seem to confirm this. The flux tube interpretation of coronal loops suggests that most loops should expand with height above the photosphere, since flux tube crosssectional area and magnetic field strength are inversely proportional. This expansion, defined by

$\Gamma=\frac{r_{\mathrm{a}}}{r_{\mathrm{f}}}$

where $r_{\mathrm{a}}$ is the radius at the apex and $r_{\mathrm{f}}$ is the radius at the footpoint (see e.g., Klimchuk 2000), has been estimated for a number of loops. Analysing Yohkoh data, Klimchuk (2000) measured a median value of $\Gamma \approx 1.30$ for soft $\mathrm{X}$-ray loops. Using TRACE data, Watko \& Klimchuk (2000) measured mean values of 1.16 and 1.20 for nonflare and postflare EUV loops, respectively. So far, potential and force-free magnetic field extrapolations tend to predict larger loop expansions than those observed (see e.g., McClymont \& Mikic et al. 1994; Gary 1996; Lopéz Fuentes et al. 2006; Klimchuk et al. 2000). There have been a number of ideas proposed to explain this. One interpretation is based on the fact that resolution of previous/current imagers have simply not been sufficient to observe coronal loop expansion accurately enough (DeForest 2007). However, Lopéz Fuentes et al. (2007) strongly disagree with this interpretation and suggest that the observed near constancy of loop width is due to the magnetic field within loops being highly tangled. Whatever the proposed magnetic structure of a coronal loop may be, e.g., potential or a more complex current carrying tangled magnetic field, this must also be consistent with its observed oscillatory properties. It is the purpose of this paper to look at the simplest of these scenarios and predict the oscillatory properties of a loop that expands with height above the photosphere embedded in a potential field.

There have been previous studies of longitudinal and transversal MHD wave propagation along thin magnetic flux tubes with variable cross sectional area embedded in a nonmagnetic environment (see e.g., Roberts \& Webb 1978; Spruit 1981; Rae \& Roberts 1982). However, in the corona it is more realistic to model a loop as an expanding magnetic flux tube embedded in a magnetic environment. An initial study into the effect of a variable longitudinal magnetic field in a flux tube embedded in a magnetic environment was done by V07. In that paper Verth considered a thin flux tube where the magnetic field, to zeroth order was taken to be purely axial, similar to the approximation of e.g., Roberts \& Webb (1978). Interestingly, it was found that the effect of magnetic field strength decreasing with height has the opposite effect on amplitude profiles and frequency mode ratios to that of gravitational density stratification. It is the purpose of this paper to follow on from the work of V07, to model an expanding flux tube (referred to hereafter as the EFT model) so that the effect of the observable expansion factor $\Gamma$, along with density stratification can be quantified.

\section{Magnetic field equilibrium}

\section{1. $2 D$ potential field}

Using cylindrical coordinates $(r, \theta, z)$, a magnetic flux tube of length $2 L$ is modelled with arbitrary internal and external longitudinal densities $\rho_{\mathrm{i}}(z)$ and $\rho_{\mathrm{e}}(z)$. To model a magnetic field equilibrium that decreases in strength with height above the photosphere, an expanding flux tube is constructed with rotational

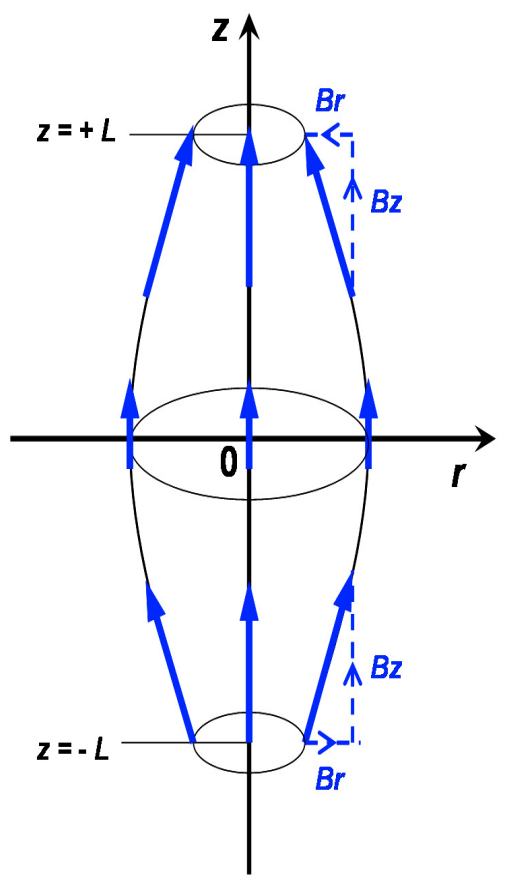

Fig. 1. The equilibrium magnetic field of an expanding flux tube.

symmetry about the $z$-axis (see Fig. 1). Therefore it is required that

$\boldsymbol{B}=B_{r}(r, z) \boldsymbol{e}_{r}+B_{z}(r, z) \boldsymbol{e}_{z}$

so that the solenoidal condition,

$\nabla \cdot \boldsymbol{B}=0$

is satisfied. Since the purpose of this paper is to investigate the fast kink mode only, we neglect gravity and consider the plasma to be cold. Hence, the magnetic field is only required to be in equilibrium with itself. Choosing a potential field configuration, magneto-static equilibrium demands that

$\nabla \times \boldsymbol{B}=\mathbf{0}$

must also be satisfied. By the equilibrium field given in Eq. (2), Eq. (4) is equivalent to

$\frac{\partial B_{r}}{\partial z}-\frac{\partial B_{z}}{\partial r}=0$

For convenience we shall use the vector potential $\boldsymbol{A}$, defined by

$\boldsymbol{B}=\nabla \times \boldsymbol{A}$,

which automatically satisfies condition (3). To find the required form of the magnetic field $\boldsymbol{B}$ given by (2) that also satisfies condition (4), we define $\boldsymbol{A}$ with an azimuthal component only such that,

$\boldsymbol{A}=\frac{\psi(r, z)}{r} \boldsymbol{e}_{\theta}$

The vector potential described by (7) is convenient since $\psi$ is constant along field lines (see e.g., Browning \& Priest 1982). In terms of $\psi$, the components of $\boldsymbol{B}$ are now given by

$B_{r}=-\frac{1}{r} \frac{\partial \psi}{\partial z}$ 
and

$B_{z}=\frac{1}{r} \frac{\partial \psi}{\partial r}$

The force-free condition described by Eq. (4) is now equivalent to

$\frac{\partial^{2} \psi}{\partial r^{2}}-\frac{1}{r} \frac{\partial \psi}{\partial r}+\frac{\partial^{2} \psi}{\partial z^{2}}=0$

By inspection, one possible solution to Eq. (10) is

$\psi=\left(C_{1}+C_{2} z\right) r^{2}+C_{3} z+C_{4}$,

where all $C_{n}$ are arbitrary constants. The method of separation of variables is also used to find a particular solution to Eq. (10). Defining $\psi=R(r) Z(z)$, Eq. (10) is equivalent to

$\frac{\mathrm{d}^{2} Z}{\mathrm{~d} z^{2}}-\kappa^{2} Z=0$

and

$\frac{\mathrm{d}^{2} R}{\mathrm{~d} r^{2}}-\frac{1}{r} \frac{\mathrm{d} R}{\mathrm{~d} r}+\kappa^{2} R=0$,

where $\kappa^{2}$ is the separation constant. For the longitudinal domain $|z| \leq L$, we must have $\kappa^{2}>0$ to have a finite bounded solution in the radial direction. It must be noted at this point, that the choice of $\kappa^{2}>0$ is arbitrary and will determine the nature of the solution. Here, we have chosen to normalise the $r$ and $z$ spatial scales to loop half length $L$, therefore we define $\kappa$ as

$\kappa=\frac{1}{L}$.

The general solutions to Eqs. (12) and (13) with scaling (14) are

$Z=C_{5} \cosh \left(\frac{z}{L}\right)+C_{6} \sinh \left(\frac{z}{L}\right)$

and

$R=r\left[C_{7} J_{1}\left(\frac{r}{L}\right)+C_{8} Y_{1}\left(\frac{r}{L}\right)\right]$,

where $J_{1}$ and $Y_{1}$ are Bessel functions of the first and second kind. Hence, combining Eqs. (11), (15) and (16) gives the general solution to the force-free Eq. (10) as follows,

$$
\begin{aligned}
\psi= & \left(C_{1}+C_{2} z\right) r^{2}+C_{3} z+C_{4}+r\left[C_{5} \cosh \left(\frac{z}{L}\right)+C_{6} \sinh \left(\frac{z}{L}\right)\right] \\
& \times\left[C_{7} J_{1}\left(\frac{r}{L}\right)+C_{8} Y_{1}\left(\frac{r}{L}\right)\right] .
\end{aligned}
$$

From the definition of $B_{r}$ given by Eqs. (8) and (17) it follows that

$$
\begin{aligned}
B_{r}= & -C_{2} r-\frac{C_{3}}{r}-\frac{1}{L}\left[C_{5} \sinh \left(\frac{z}{L}\right)+C_{6} \cosh \left(\frac{z}{L}\right)\right] \\
& \times\left[C_{7} J_{1}\left(\frac{r}{L}\right)+C_{8} Y_{1}\left(\frac{r}{L}\right)\right] .
\end{aligned}
$$

It is required that $B_{r}$ is bounded on $r$ and is an odd function about $z=0$ (see Fig. 1) so we must have $C_{2}=C_{3}=C_{6}=C_{8}=0$. It is also required that $J_{1}(r / L)$ does not change sign on the physically relevant $r$ domain. Since in Sect. 4, the aim is to describe only linear perturbations of the tube, the required $r$ range is from $r=0$ to $r \approx r_{0}$, where $r_{0}$ is the tube boundary. By observation $r_{0} / L \ll$ 1 , therefore $r_{0} / L \ll j_{1,1} \approx 3.83$ (the first zero of $J_{1}$ ). Hence on the $r$ domain of interest, $J_{1} \geq 0$ as required. Let $A_{1}=C_{5} C_{7}$, then the expression for $B_{r}$ can be simplified to

$B_{r}=-\frac{A_{1}}{L} \sinh \left(\frac{z}{L}\right) J_{1}\left(\frac{r}{L}\right)$

The chosen direction of the magnetic field shown in Fig. 1 requires that $B_{r} \geq 0$ for $z \leq 0$ which implies that $A_{1}>0$. We now check that the chosen form of $\psi$ also gives the required functional form of $B_{z}$. Using Eq. (9) and the well known relation that

$J_{v}{ }^{\prime}(x)=J_{v-1}(x)-\frac{v}{x} J_{v}(x)$

for all $v$ and $x$ (see e.g., Abramowitz \& Stegun 1965), it can be easily calculated that

$B_{z}=A_{2}+\frac{A_{1}}{L} \cosh \left(\frac{z}{L}\right) J_{0}\left(\frac{r}{L}\right)$

where $A_{2}=2 C_{1}$. By inspection of Fig. 1 , it is required that $B_{z}$ is a positive even function about $z=0$ and is monotonically increasing as $z \rightarrow \pm L$. Since $r_{0} / L \ll j_{0,1} \approx 2.40$ (the first zero of $J_{0}$ ), we have that $J_{0}>0$ on the physically relevant $r$ domain. Hence, Eq. (21) satisfies these all these requirements if $A_{2}>$ $-A_{1} / L$. The constant $C_{4}$ in Eq. (17) is not required for the present study, therefore Eq. (17) can be simplified to

$\psi=\frac{A_{2}}{2} r^{2}+A_{1} r \cosh \left(\frac{z}{L}\right) J_{1}\left(\frac{r}{L}\right)$

\subsection{Equilibrium quantities at the tube boundary}

The purpose of this section is to derive explicit expressions for $r$, $B_{r}$ and $B_{z}$ at the tube boundary which depend on $z$ only. These expressions will then be used in the governing radial velocity equation for the fast kink body mode in Sect. 4. The thin flux tube parameter is defined as follows,

$\epsilon_{1}=\frac{r_{\mathrm{a}}}{L}$

Denoting the $r$ and $z$ components of the magnetic field at the footpoints as

$B_{r}\left(r_{\mathrm{f}}, \pm L\right)=\mp B_{r, \mathrm{f}}$

and

$B_{z}\left(r_{\mathrm{f}}, \pm L\right)=B_{z, \mathrm{f}}$,

where $B_{r, \mathrm{f}} \geq 0$ and $B_{z, \mathrm{f}}>0$, one can then define the constants $A_{1}$ and $A_{2}$ in Eqs. (19) and (21) in terms of physical parameters as follows,

$A_{1}=\frac{B_{r, \mathrm{f}} L}{\sinh (1) J_{1}\left(\epsilon_{1} / \Gamma\right)}$

and

$A_{2}=B_{z, \mathrm{f}}-\frac{B_{r, \mathrm{f}} J_{0}\left(\epsilon_{1} / \Gamma\right)}{\tanh (1) J_{1}\left(\epsilon_{1} / \Gamma\right)}$.

By observation, $\epsilon_{1} \ll 1$ and we also assume that the small expansion observed by Klimchuk (2000) and Watko \& Klimchuk (2000) were of fully resolved loops so that $\Gamma \approx 1$. Then the Bessel function terms in Eqs. (26) and (27) can be approximated to first order as

$J_{0}\left(\frac{\epsilon_{1}}{\Gamma}\right) \approx 1$ 
and

$J_{1}\left(\frac{\epsilon_{1}}{\Gamma}\right) \approx \frac{\epsilon_{1}}{2 \Gamma}$.

Since $\Gamma \approx 1$, it follows that $B_{r} \ll B_{z}$, so we must also introduce the small parameter $\epsilon_{2}$ such that

$\epsilon_{2}=\frac{B_{r, \mathrm{f}}}{B_{z, \mathrm{f}}}$

By magnetic flux conservation,

$\Gamma^{2}=\frac{B_{\mathrm{f}}}{B_{a}}$

and relation (30) we have that

$\Gamma^{2} \approx \frac{B_{z, \mathrm{f}}}{B_{z, \mathrm{a}}}$

Using the expression for $B_{z}$ given by Eqs. (21), (26), (27), definition (30) and approximations (28), (29) in Eq. (32) gives the following relation between the small parameters, $\epsilon_{1}$ and $\epsilon_{2}$,

$\frac{\epsilon_{2}}{\epsilon_{1}} \approx \frac{\left(1-\Gamma^{2}\right)}{2 \Gamma^{3}} \frac{\sinh (1)}{1-\cosh (1)}$.

For $\Gamma \geq 1$, Eq. (33) shows that the magnetic equilibrium has $\epsilon_{2}<\epsilon_{1}$. Since $r_{0} / L \ll 1$ we also have that

$J_{1}\left(\frac{r_{0}}{L}\right) \approx \frac{r_{0}}{2 L}$.

Using the approximations (28), (29), (34), Eqs. (22), (26), (27), (33) and definition (30), $\psi$ at the flux tube boundary is given by

$\psi\left(r_{0}, z\right) \approx \frac{B_{z, \mathrm{f}} r_{0}^{2}}{2}\left\{1+\frac{\left(1-\Gamma^{2}\right)}{\Gamma^{2}} \frac{\left[\cosh \left(\frac{z}{L}\right)-\cosh (1)\right]}{1-\cosh (1)}\right\}$.

By the choice of vector potential (7), the magnetic surface denoting the boundary of the flux tube has a constant $\psi$ value. Using Eq. (35), at the loop footpoints the boundary value of $\psi$ is given by

$\psi\left(r_{\mathrm{f}}, \pm L\right) \approx \frac{B_{z, \mathrm{f}} r_{\mathrm{f}}^{2}}{2}$.

Hence using Eqs. (35) and (36), we obtain an explicit expression for the radius of the flux tube boundary as a function of $z$ given by

$r_{0}(z) \approx r_{\mathrm{f}}\left\{1+\frac{\left(1-\Gamma^{2}\right)}{\Gamma^{2}} \frac{\left[\cosh \left(\frac{z}{L}\right)-\cosh (1)\right]}{1-\cosh (1)}\right\}^{-\frac{1}{2}}$.

Similarly, $B_{r}$ and $B_{z}$ at the tube boundary can also be described explicitly as functions of $z$,

$B_{r}(z) \approx-B_{z, \mathrm{f}} \frac{\left(1-\Gamma^{2}\right)}{2 \Gamma^{2}} \frac{\sinh \left(\frac{z}{L}\right)}{1-\cosh (1)} \frac{r_{0}(z)}{L}$

and

$B_{z}(z) \approx B_{z, \mathrm{f}}\left\{1+\frac{\left(1-\Gamma^{2}\right)}{\Gamma^{2}} \frac{\left[\cosh \left(\frac{z}{L}\right)-\cosh (1)\right]}{1-\cosh (1)}\right\}$.

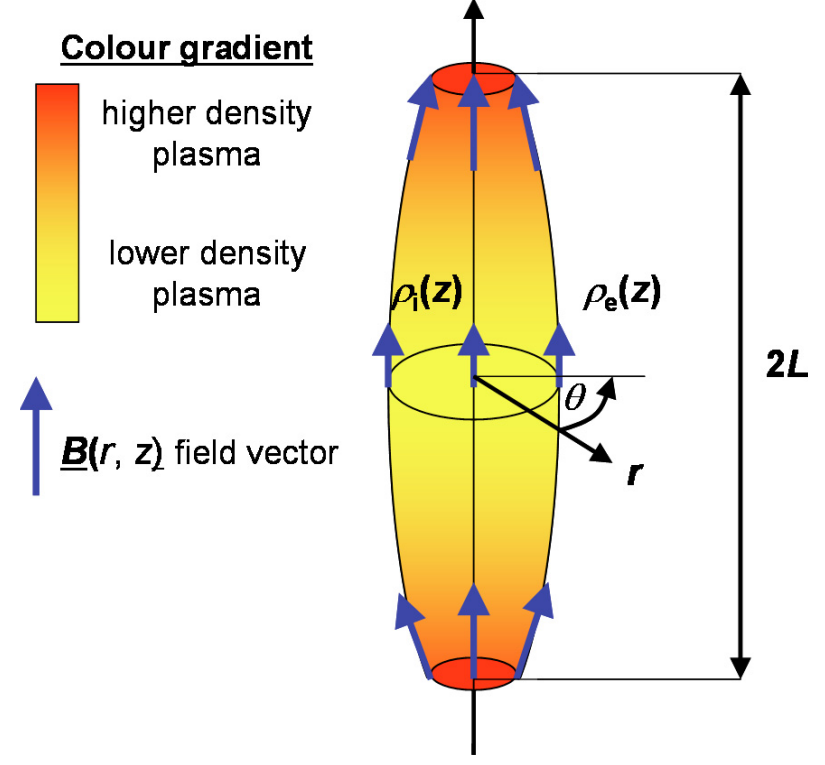

Fig. 2. Stratification of plasma density and inhomogeneous magnetic field.

\section{Linearising the MHD equations}

The cold and ideal MHD equations are linearised by assuming small perturbations of the magnetic field, $\boldsymbol{b}=\left(b_{r}, b_{\theta}, b_{z}\right)$ about the force-free magnetic equilibrium (2) and velocity perturbations, $\boldsymbol{v}=\left(v_{r}, v_{\theta}, v_{z}\right)$ about a plasma in static equilibrium, resulting in the following system of equations,

$\mu \rho \frac{\partial v_{r}}{\partial t}=B_{z}\left(\frac{\partial b_{r}}{\partial z}-\frac{\partial b_{z}}{\partial r}\right)$

$\mu \rho \frac{\partial v_{\theta}}{\partial t}=\mathcal{D}_{\|} b_{\theta}-\frac{\mu}{r} \frac{\partial P}{\partial \theta}$

$\mu \rho \frac{\partial v_{z}}{\partial t}=-B_{r}\left(\frac{\partial b_{r}}{\partial z}-\frac{\partial b_{z}}{\partial r}\right)$

$\frac{\partial b_{r}}{\partial t}=-\frac{B_{r}}{r} \frac{\partial v_{\theta}}{\partial \theta}+\frac{\partial}{\partial z}\left(B_{z} v_{r}-B_{r} v_{z}\right)$,

$\frac{\partial b_{z}}{\partial t}=-\frac{1}{r} \frac{\partial}{\partial r}\left[r\left(B_{z} v_{r}-B_{r} v_{z}\right)\right]-\frac{B_{z}}{r} \frac{\partial v_{\theta}}{\partial \theta}$.

and

$\frac{1}{r} \frac{\partial}{\partial r}\left(r b_{r}\right)+\frac{1}{r} \frac{\partial b_{\theta}}{\partial \theta}+\frac{\partial b_{z}}{\partial z}=0$,

where in Eq. (41) operator $\mathcal{D}_{\|}$is defined as

$\mathcal{D}_{\|} \equiv B_{r} \frac{\partial}{\partial r}+B_{z} \frac{\partial}{\partial z}$

and $P$ is the total perturbation to magnetic pressure,

$P=\frac{\boldsymbol{B} \cdot \boldsymbol{b}}{\mu}$.

Note that Eqs. (40) and (42) have been simplified using Eq. (5). From the equilibrium magnetic field shown in Fig. 1, it is clear that perturbations normal to the surface of the tube will have 
both $r$ and $z$ components. From Eqs. (40) and (42), it follows that

$\frac{\partial v_{z}}{\partial t}=-\frac{B_{r}}{B_{z}} \frac{\partial v_{r}}{\partial t}$

Since the equilibrium quantities only depend on $r$ and $z$, we can introduce the following Fourier decomposition

$f(r, \theta, z)=\hat{f}(r, z) \mathrm{e}^{\mathrm{i}(m \theta-\omega t)}$

where $f$ is any perturbed quantity, $m$ is the azimuthal wave number (a positive integer) and $\omega$ is the angular frequency of the oscillations. Then Eq. (48) becomes

$\hat{v}_{z}=-\frac{B_{r}}{B_{z}} \hat{v}_{r}$

Since $B_{r} \ll B_{z}$, Eq. (50) shows the $z$ component of the velocity perturbation is only a small correction to the total perturbation. Therefore, in the following analysis we shall concentrate primarily on the dominant $r$ component. Taking the time derivative of Eq. (40), substituting Eqs. (43) and (44) and using the relation (50) results in the following equation which describes the $r$ component of plasma motion,

$$
\begin{aligned}
\frac{\mu \rho}{B_{z}} \frac{\partial^{2} v_{r}}{\partial t^{2}}= & \frac{\partial^{2}}{\partial z^{2}}\left(\frac{B^{2}}{B_{z}} v_{r}\right)-\frac{\partial^{2} b_{z}}{\partial r \partial t} \\
& +\frac{\partial}{\partial z}\left\{\frac{B_{r}}{B_{z}}\left[\frac{\partial b_{z}}{\partial t}+\frac{1}{r} \frac{\partial}{\partial r}\left(\frac{B^{2}}{B_{z}} r v_{r}\right)\right]\right\} .
\end{aligned}
$$

Equation (51) shows that we must know $b_{z}$ before we can find the velocity perturbation $v_{r}$. Taking the time derivative of Eq. (44) and substituting Eqs. (40)-(42) and (45) results in the following equation which describes the $z$ component of the magnetic field perturbation,

$$
\begin{aligned}
\frac{\partial^{2} b_{z}}{\partial t^{2}}=- & \frac{1}{r} \frac{\partial}{\partial r}\left[r \frac{B^{2}}{\mu \rho}\left(\frac{\partial b_{r}}{\partial z}-\frac{\partial b_{z}}{\partial r}\right)\right] \\
& +\frac{B_{z}}{\mu \rho} \frac{1}{r}\left[\mathcal{D}_{\|}\left(\frac{\partial}{\partial r}\left(r b_{r}\right)+r \frac{\partial b_{z}}{\partial z}\right)+\frac{\mu}{r} \frac{\partial^{2} P}{\partial \theta^{2}}\right],
\end{aligned}
$$

where $B^{2}=B_{r}^{2}+B_{z}^{2}$. Equation (52) shows that the $z$ and $r$ components of the magnetic field perturbations are coupled but it will be shown in the following section that the observed expansion factor, $\Gamma \approx 1$ and relations $r_{0} \ll L$ and $B_{r} \ll B_{z}$ effectively decouple these perturbations for the fast kink body mode.

\section{Governing equation for fast kink body mode}

The fast kink mode is essentially incompressible with $b_{z} \ll b_{r}$. In the thin flux tube limit, $b_{z} / b_{r} \leq \epsilon_{1}$, therefore the scaling $\tilde{b}_{r}=\epsilon_{1} b_{r}$ is introduced. By Eq. (30), $B_{r} / B_{z} \leq \epsilon_{2}$, accordingly, the radial magnetic field component is rescaled with $B_{r}=\epsilon_{2} \tilde{B}_{r}$. Inside the tube, the characteristic scale in the radial direction is $r_{0}$ but outside the tube it is assumed to be $L$, therefore the stretching coordinate $\zeta=\epsilon_{1} z$ is introduced in the axial direction both inside and outside the tube. Consequently, the stretching coordinate $\xi=\epsilon_{1} r$ rescales the radial direction outside the tube. This implies that the frequency both outside and inside the tube becomes $\tilde{\omega}=\omega / \epsilon_{1}$. Applying Fourier decomposition given by Eq. (49) with $m=1$ (the observed kink mode) and assuming small tube expansion such that $\epsilon_{2}<\epsilon_{1}^{2}$, the $r$ and $z$ magnetic perturbations are effectively decoupled and Eq. (52) can be approximated by

$\frac{\partial}{\partial r}\left(r \frac{\partial \hat{b}_{z}}{\partial r}\right)-\frac{\hat{b}_{z}}{r}=0$

inside the tube and

$\frac{1}{\xi} \frac{\partial}{\partial \xi}\left(\xi \frac{\partial \hat{b}_{z}}{\partial \xi}\right)+\frac{\partial^{2} \hat{b}_{z}}{\partial \zeta^{2}}+\left(\frac{\tilde{\omega}^{2}}{v_{A, \mathrm{e}}^{2}}-\frac{1}{\xi^{2}}\right) \hat{b}_{z}=0$

outside the tube, where $v_{A, \mathrm{e}}^{2}=B_{z}^{2} /\left(\mu \rho_{\mathrm{e}}\right)$. Note that the potential field relationship between $B_{r}$ and $B_{z}$ shown in Eq. (5) has also been used to simplify Eqs. (53) and (54). The solution of Eq. (53), regular at $r=0$ is

$\hat{b}_{z}=A_{\mathrm{i}}(\zeta) r$.

Equation (54) is similar in form to that derived by Dymova \& Ruderman (2005) (for the case when $B_{z}$ is constant) and can be solved using separation of variables and an asymptotic expansion near the tube boundary (see Dymova \& Ruderman 2005, for details), giving the solution outside the tube,

$\hat{b}_{z}=A_{\mathrm{e}}(\zeta) r^{-1}$.

The solutions for $\hat{b}_{z}$ given by (55) and (56) can be substituted in Eq. (51), giving the governing equation for radial velocity inside the tube as

$\frac{\partial^{2}}{\partial \zeta^{2}}\left(B_{z} \hat{v}_{r, \mathrm{i}}\right)+\frac{\partial}{\partial \zeta}\left[\frac{\tilde{B}_{r}}{B_{z}} \frac{1}{r} \frac{\partial}{\partial r}\left(r B_{z} \hat{v}_{r, \mathrm{i}}\right)\right]+\left(\frac{\tilde{\omega}}{v_{A, i}}\right)^{2} B_{z} \hat{v}_{r, \mathrm{i}}=-\frac{\mathrm{i} \tilde{\omega}}{\epsilon_{1}} A_{\mathrm{i}}$

and outside as

$\frac{\partial^{2}}{\partial \zeta^{2}}\left(B_{z} \hat{v}_{r, \mathrm{e}}\right)+\left(\frac{\tilde{\omega}}{v_{A, \mathrm{e}}}\right)^{2} B_{z} \hat{v}_{r, \mathrm{e}}=\frac{\mathrm{i} \tilde{\omega}}{\epsilon_{1}} \frac{A_{\mathrm{e}}}{r^{2}}$

By inspection, we look for solutions to Eqs. (57) and (58) in the following form,

$B_{z} \hat{v}_{r, i}=G_{\mathrm{i}}(\zeta)$

and

$B_{z} \hat{v}_{r, e}=G_{\mathrm{e}}(\zeta) r^{-2}$.

Substituting Eqs. (59) and (60) in Eqs. (57) and (58), respectively, results in

$\frac{\mathrm{d}^{2} G_{\mathrm{i}}}{\mathrm{d} \zeta^{2}}+\frac{1}{r} \frac{\partial}{\partial \zeta}\left(\frac{\tilde{B}_{r}}{B_{z}} G_{\mathrm{i}}\right)+\left(\frac{\tilde{\omega}}{v_{A, \mathrm{i}}}\right)^{2} G_{\mathrm{i}}=-\frac{\mathrm{i} \tilde{\omega}}{\epsilon_{1}} A_{\mathrm{i}}$

and

$\frac{\mathrm{d}^{2} G_{\mathrm{e}}}{\mathrm{d} \zeta^{2}}+\left(\frac{\tilde{\omega}}{v_{A, \mathrm{e}}}\right)^{2} G_{\mathrm{e}}=\frac{\mathrm{i} \tilde{\omega}}{\epsilon_{1}} A_{\mathrm{e}}$

Returning to the initial longitudinal $z$ variable, to match solutions (61) and (62) at the tube boundary $r=r_{0}$ (where all quantities are functions of $z$ only), the continuity of $\hat{b}_{z}$ and $\hat{v}_{r}$ requires that

$A_{\mathrm{e}}=A_{\mathrm{i}} r_{0}^{2}$

and

$G_{\mathrm{e}}=G_{\mathrm{i}} r_{0}^{2}$. 
Substituting Eqs. (63) and (64) into Eq. (62) and adding the resulting equation to (61), one arrives at

$$
\begin{aligned}
G_{\mathrm{i}}^{\prime \prime} & +\frac{1}{2 r_{0}}\left(\frac{B_{r}}{B_{z}}+4 r_{0}^{\prime}\right) G_{\mathrm{i}}^{\prime} \\
& +\left[\left(\frac{\omega}{c_{k}}\right)^{2}+\frac{1}{2 r_{0}}\left(\frac{B_{r}}{B_{z}}\right)^{\prime}+\left(\frac{r_{0}^{\prime}}{r_{0}}\right)^{2}+\frac{r_{0}^{\prime \prime}}{r_{0}}\right] G_{\mathrm{i}}=0,
\end{aligned}
$$

where' $\equiv \mathrm{d} / \mathrm{d} z$ and

$c_{k}^{2}=\frac{2 B_{z}^{2}}{\mu\left(\rho_{\mathrm{i}}+\rho_{\mathrm{e}}\right)}$

is the fast kink speed. Dispensing with the $i$ subscript notation and substituting Eq. (59) into Eq. (65), the governing equation for the fast kink body mode in terms of radial velocity at the tube boundary is

$$
\begin{aligned}
& \left(B_{z} \hat{v}_{r}\right)^{\prime \prime}+\frac{1}{2 r_{0}}\left(\frac{B_{r}}{B_{z}}+4 r_{0}^{\prime}\right)\left(B_{z} \hat{v}_{r}\right)^{\prime} \\
& +\left[\left(\frac{\omega}{c_{k}}\right)^{2}+\frac{1}{2 r_{0}}\left(\frac{B_{r}}{B_{z}}\right)^{\prime}+\left(\frac{r_{0}^{\prime}}{r_{0}}\right)^{2}+\frac{r_{0}^{\prime \prime}}{r_{0}}\right] B_{z} \hat{v}_{r}=0 .
\end{aligned}
$$

In the case of a flux tube with constant $B_{z}$ and arbitrary longitudinal density stratification, Eq. (67) simply reduces to

$\hat{v}_{r}^{\prime \prime}+\left(\frac{\omega}{c_{k}}\right)^{2} \hat{v}_{r}=0$

which is in agreement with Dymova \& Ruderman (2005) and Erdélyi \& Verth (2007). The solutions to Eq. (68) have been extensively studied by Dymova \& Ruderman (2005), Erdélyi \& Verth (2007) and Verth et al. (2007). Substituting the expressions for equilibrium quantities from Eqs. (37)-(39), Eq. (67) is equivalent to

$f_{2}(z) \hat{v}_{r}^{\prime \prime}+f_{1}(z) \hat{v}_{r}^{\prime}+f_{0}(z) \hat{v}_{r}=0$,

where functions $f_{n}(z)$ are

$$
\begin{aligned}
f_{0}(z)= & a_{6} \cosh ^{2}\left(\frac{z}{L}\right)+a_{7} \cosh \left(\frac{z}{L}\right)+a_{8} \\
& +a_{9}\left[\frac{\mu\left(\rho_{\mathrm{i}}+\rho_{\mathrm{e}}\right) \omega}{B_{z, \mathrm{f}}}\right]^{2}, \\
f_{1}(z)= & \sinh \left(\frac{z}{L}\right)\left[a_{4} \cosh \left(\frac{z}{L}\right)+a_{5}\right], \\
f_{2}(z)= & a_{1} \cosh ^{2}\left(\frac{z}{L}\right)+a_{2} \cosh \left(\frac{z}{L}\right)+a_{3},
\end{aligned}
$$

and $a_{n}$ are the following constants,

$$
\begin{aligned}
& a_{1}=8 L^{2}\left(\Gamma^{4}-2 \Gamma^{2}+1\right), \\
& a_{2}=-16 L^{2}\left(\Gamma^{4}-c \Gamma^{2}+c\right), \\
& a_{3}=8 L^{2}\left(\Gamma^{4}-2 c \Gamma^{2}+c^{2}\right), \\
& a_{4}=6 L\left(\Gamma^{4}-2 \Gamma^{2}+1\right), \\
& a_{5}=-6 L\left[\Gamma^{4}-(c+1) \Gamma^{2}+c\right], \\
& a_{6}=3\left(\Gamma^{4}-2 \Gamma^{2}+1\right), \\
& a_{7}=-2\left[\Gamma^{4}-(c+1) \Gamma^{2}+c\right], \\
& a_{8}=-\left(\Gamma^{4}-2 \Gamma^{2}+1\right), \\
& a_{9}=8 L^{2} \Gamma^{4}\left(c^{2}-2 c+1\right), \\
& \text { with } c=\cosh (1) .
\end{aligned}
$$

\section{Flux tube expansion with constant density}

The solutions to Eq. (69) will depend on the parameter $\Gamma$ and the functional forms chosen for $\rho_{\mathrm{i}}$ and $\rho_{\mathrm{e}}$ in Eq. (70). Since previous studies have quantified the effect of density stratification with a constant magnetic field, it will be instructive here to study the effect of magnetic stratification with constant density. We know of no analytical solution to equations of general type (69) when $\rho_{\mathrm{i}}$ and $\rho_{\mathrm{e}}$ are chosen to be arbitrary constants. However, transforming Eq. (69) to its canonical form

$u^{\prime \prime}+f u=0$,

where

$f=f_{0}-\frac{1}{4}\left(\frac{f_{1}}{f_{2}}\right)^{2}-\frac{1}{2}\left(\frac{f_{1}}{f_{2}}\right)^{\prime}$

and

$\hat{v}_{r}=u(z) \exp \left(-\frac{1}{2} \int \frac{f_{1}}{f_{2}} \mathrm{~d} z\right)$,

it is found that Eq. (82) with constant densities $\rho_{\mathrm{i}}$ and $\rho_{\mathrm{e}}$ can be very closely approximated by the Halm equation (see e.g., Polyanin \& Zaitsev 2003) where

$f=f_{a}\left[1+\left(\sqrt{\frac{f_{a}}{f_{\mathrm{f}}}}-1\right)\left(\frac{z}{L}\right)^{2}\right]^{-2}$,

$f_{a}=f(0)$ and $f_{f}=f( \pm L)$. The general solution to Eq. (82), where $f$ is described by Eq. (85), is

$u(z)=\sqrt{\alpha^{2}+1}$

$$
\times\left\{C_{n} \cos [\eta \arctan (\alpha)]+D_{n} \sin [\eta \arctan (\alpha)]\right\},
$$

where $C_{n}$ and $D_{n}$ are arbitrary constants,

$\alpha=\delta \frac{z}{L}$,

$\delta^{2}=\sqrt{\frac{f_{a}}{f_{\mathrm{f}}}}-1$

and

$\eta=\sqrt{\frac{f_{a}}{L} \delta+1}$.

To find the standing mode solution to Eq. (82), it is required that

$u( \pm L)=0$.

Let $n=1,2,3, \ldots$ correspond to the mode number. Then by Eqs. (86) and (90), the dispersion relation is given by the following transcendental equation,

$\eta \arctan (\delta)=\frac{n \pi}{2}$.

Dispersion relation (91) can be solved asymptotically, since for $\Gamma \approx 1$, the following approximations can be made,

$f_{a} \approx\left(\frac{\omega_{n}}{c_{k, \mathrm{f}}}\right)^{2}$

and

$\delta^{2} \approx \Gamma^{2}-1$, 


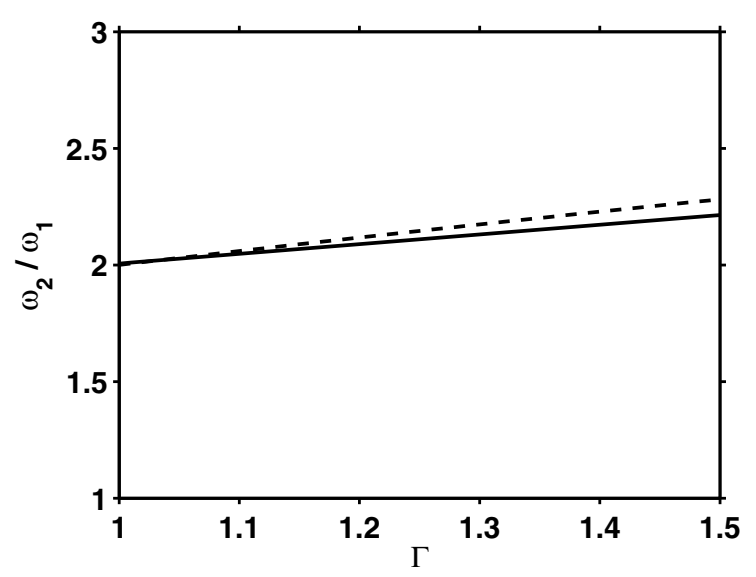

Fig. 3. Frequency ratio $\omega_{2} / \omega_{1}$ against $\Gamma$. Solid line is numerically derived from governing equation, Eq. (69) and the dashed line shows the analytically derived relation given by Eq. (95).

where $c_{k, \mathrm{f}}$ is the kink speed at the footpoints. Applying asymptotic approximations (92) and (93) along with Eqs. (89) to (91), results in an explicit expression for the mode frequency,

$\left(\frac{\omega_{n}}{c_{k, \mathrm{f}}}\right)^{2} \approx \frac{L}{\sqrt{\Gamma^{2}-1}}\left[\left(\frac{n \pi}{2 \arctan \left(\sqrt{\Gamma^{2}-1}\right)}\right)^{2}-1\right]$.

Hence by Eq. (94), the ratio of the first harmonic $(n=2)$ to the fundamental mode $(n=1)$ squared is

$\left(\frac{\omega_{2}}{\omega_{1}}\right)^{2} \approx \frac{\pi^{2}-\arctan ^{2}\left(\sqrt{\Gamma^{2}-1}\right)}{(\pi / 2)^{2}-\arctan ^{2}\left(\sqrt{\Gamma^{2}-1}\right)}$.

Equation (95) shows that change in frequency ratio is purely dependent on the loop expansion factor $\Gamma$. Hence by Eq. (95), for a loop with constant density, the lowest order correction due to magnetic stratification is

$\frac{\omega_{2}}{\omega_{1}} \approx 2\left[1+\frac{3\left(\Gamma^{2}-1\right)}{2 \pi^{2}}\right]$.

Equation (96) clearly shows that the frequency ratio $\omega_{2} / \omega_{1}>$ 2 for an expanding magnetic flux tube with constant density. Therefore, if magnetic field strength is decreasing with height above the photosphere, this has the opposite effect to that of density stratification (cf. Andries et al. 2005a), in agreement with V07. The relationship between $\omega_{2} / \omega_{1}$ and $\Gamma$ given by Eq. (95) is compared with values derived numerically, by solving Eq. (69) using the shooting method (shown in Fig. 3). When $\Gamma=1.3$, the solutions only differ by a few percent, hence Eq. (95) is a very reasonable analytical approximation of the frequency ratio $\omega_{2} / \omega_{1}$.

Using Eq. (3) of V07 and the equilibrium magnetic field given by Eq. (39) in this paper it can be shown that the frequency ratio results with the purely axial field approximation and EFT models are in excellent agreement. Hence the eigenvalues of the EFT governing Eq. (67) correspond to those of the much simpler Eq. (3) in V07. However, with increasing magnetic stratification a difference becomes apparent in the amplitude profiles (see comparison in Fig. 4). This would be relevant if one wanted to attempt spatial magneto-seismology as suggested by Erdélyi \& Verth (2007) and Verth et al. (2007).

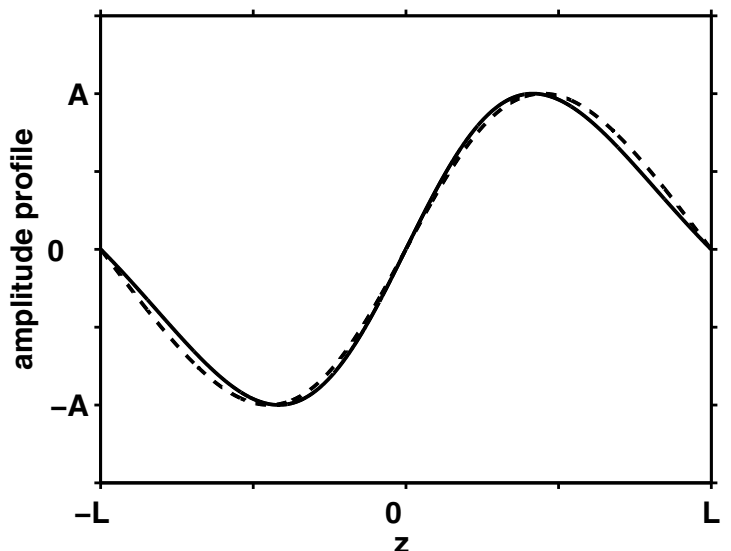

Fig. 4. Amplitude profile of the 1st harmonic plotted against $z(\Gamma=1.3)$ for the EFT (solid line) and V07 (dashed line) models.

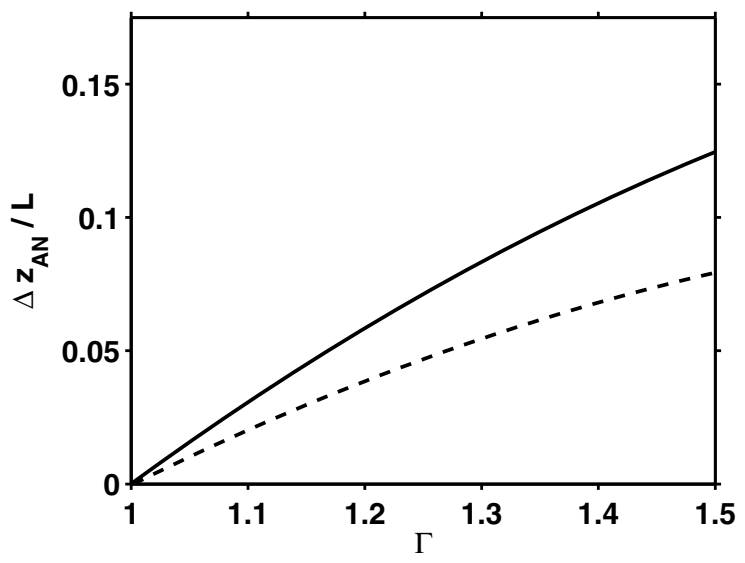

Fig. 5. Normalised shift of 1st harmonic anti-node plotted against $\Gamma$ for the EFT (solid line) and V07 (dashed line) models.

This difference is most clearly seen if one compares the antinode shift of the first harmonic. It was pointed out by V07 that if the magnetic field becomes weaker towards the loop apex, a useful magneto-seismological signature is the anti-node of the 1st harmonic since it shifts towards the loop apex (see Fig. 4 in this paper and Fig. 5 in V07). Normalised anti-node shift $\Delta z_{\mathrm{AN}} / L$, is plotted for the two models in Fig. 5. It can be seen that the shift for the EFT model is actually greater than predicted by V07. Quantifying this by example, if we take $\Gamma=1.3$ and $L=100 \mathrm{Mm}$, the EFT model and that of V07 predict shifts of 8.24 $\mathrm{Mm}$ and 5.50 Mm, respectively, a difference of 2.74 Mm.

\section{Implications for magneto-seismology and estimating coronal density scale heights}

Interestingly, there have recently been measurements of fast kink mode loop oscillations by O'Shea et al. (2007) and De Moortel \& Brady (2007) where $\omega_{2} / \omega_{1}>2$, which may be explained by magnetic field divergence being the dominant effect over density stratification. O'Shea et al. found three examples of cool loop (temperature $\approx 0.25 \mathrm{MK}$ ) oscillations with harmonics in temporal series image data from the Coronal Diagnostic Spectrometer (CDS) onboard the SOlar and Heliospheric Observatory (SOHO). Of the three cases studied, two both had $\omega_{2} / \omega_{1} \approx 2.4$. Using the EUV $171 \AA$ passband 
(temperature $\approx 1 \mathrm{MK}$ ) of TRACE, De Moortel \& Brady analysed fast kink oscillations in a twisted S-shaped post-flare loop and identified the fundamental mode along with two higher harmonics. However, what they interpreted as the 1st harmonic is probably more consistent with the fundamental mode, as pointed out by VNV07. If this is the case then the mean value of $\omega_{2} / \omega_{1}$ is 2.11 (see Table 1 in their paper). If the interpretation of De Moortel \& Brady is indeed correct, then the mean value is 2.76 but either way, $\omega_{2} / \omega_{1}>2$. Collectively, these observations may be evidence of the importance of magnetic field divergence in governing the properties of fast kink oscillations. In specific relation to the model presented in this paper, it must be pointed out that the twisted S-shaped loop studied by De Moortel $\&$ Brady has a magnetic field structure that is far from potential. To fully understand the oscillatory properties of loops of this type, a more advanced study must be made using more general force-free fields.

V07 pointed out that neglecting the effect of magnetic stratification on loop oscillations can cause the overestimation of coronal density scale height $H$. Assuming a constant magnetic field for two oscillating loops observed with the TRACE $171 \AA$ passband, VNV07 estimated super-hydrostatic density scale heights of $H=68 \mathrm{Mm}$ and $H=109 \mathrm{Mm}$. In this passband, hydrostatic loops should have $H \approx 50 \mathrm{Mm}$, therefore a possible explanation of these estimated super-hydrostatic scale heights is that magnetic field divergence was not taken into account. The loops in question were judged to be approximately vertical to the photosphere and semi-circular. For a gravitationally stratified loop of this type the density profile is given by

$\rho_{\mathrm{i}}(z)=\rho_{\mathrm{i}, \mathrm{f}} \exp \left[-\frac{2 L}{\pi H} \cos \left(\frac{\pi z}{2 L}\right)\right]$,

where $\rho_{\mathrm{i}, \mathrm{f}}$ is the internal footpoint density. Equation (69) is solved numerically with the shooting method using the density profile (97) with $\rho_{\mathrm{e}}$ taken to be proportional to $\rho_{\mathrm{i}}$. Taking the estimates of $L$ and $\omega_{2} / \omega_{1}$ from VNV07, it is found that the super-hydrostatic scale heights of $68 \mathrm{Mm}$ and $109 \mathrm{Mm}$ can be reduced to the hydrostatic scale height with loop expansions of only $\Gamma \approx 1.1$ and $\Gamma \approx 1.5$, respectively. Hence, even the modest values of $\Gamma$ measured by Watko \& Klimchuk (2000) for TRACE EUV loops (see Figs. 14 and 15 in their paper) could prove very significant in determining an accurate value of $H$.

\section{Conclusions}

Since there is still much theoretical disagreement about the fine structure of coronal loops, it is important that any proposed models can be tested against the observed oscillatory properties. The MHD wave theory presented in this paper is an improvement on previous magnetic cylinder models of loops, in that both longitudinal plasma density and flux tube cross-sectional area (inversely proportional to magnetic field strength) are allowed to vary. It was shown in Sect. 6, that even the small loop expansion factors observed may have a significant effect on their oscillations. Hence, to conduct more precise magneto-seismology of the solar corona, magnetic field divergence should be taken into account. In the future, it will also be instructive to develop MHD wave theory which models loops with more general force-free equilibria. There are important observational challenges too in the field of coronal magneto-seismology. Since there have been so few cases of higher harmonics analysed so far, a much larger statistical study must be done before any firm conclusions can be reached about loop fine structure through the implementation of magneto-seismological techniques.

Acknowledgements. The authors thank M. Ruderman for a number of useful discussions. R.E. acknowledges M. Kéray for patient encouragement. The authors are also grateful to NSF, Hungary (OTKA, Ref. No. K67746) and the Engineering and Physical Sciences Research Council (EPSRC), UK for the financial support they received.

\section{References}

Abramowitz, M., \& Stegun, I. A. 1965, Handbook of Mathematical Functions (New York: Dover Publications)

Andries, J., Arregui, I., \& Goossens, M. 2005a, ApJ, 624, L57

Andries, J., Goossens, M., Hollweg, J. V., Arregui, I., \& Van Doorsselaere, T. 2005b, A\&A, 430, 1109

Arregui, I., Andries, J., Van Doorsselaere, T., Goossens, M., \& Poedts, S. 2007, A\&A, 463, 333

Aschwanden, M. J., De Pontieu, B., Schrijver, C. J., \& Title, A. M. 2002, Sol. Phys., 206, 99

Aschwanden, M. J., Fletcher, L., Schrijver, C. J., \& Alexander, D. 1999a, ApJ, 520,880

Aschwanden, M. J., Newmark, J. S., Delaboudinière, J., et al. 1999b, ApJ, 515, 842

Aschwanden, M. J., Nightingale, R. W., \& Alexander, D. 2000, ApJ, 541, 1059 Aschwanden, M. J., Schrijver, C. J., \& Alexander, D. 2001 ApJ, 550, 1036

Aschwanden, M. J., Nightingale, R. W., Andries, J., Goossens, M., \& Van Doorsselaere, T. 2003, ApJ, 598, 1375

Banerjee, D., Erdélyi, R., Oliver, R., \& O’Shea, E. 2007, Sol. Phys., 246, 3

Brekke, P., Kjeldseth-Moe, O., \& Harrison, R. A. 1997, Sol. Phys., 175, 511 Browning, P. K., \& Priest, E. R. 1982, Geophys. Ap. Fluid Dyn., 21, 237

Díaz, A. J., Oliver, R., \& Ballester, J. L. 2002, ApJ, 580, 550

DeForest, C. E. 2007, ApJ, 661, 532

De Moortel, I., \& Brady, C. S. 2007, ApJ, 664, 1210

Dymova, M. V., \& Ruderman, M. S. 2005, Sol. Phys., 229, 79

Dymova, M. V., \& Ruderman, M. S. 2006a, A\&A, 457, 1059

Dymova, M. V., \& Ruderman, M. S. 2006b, A\&A, 459, 241

Edwin, P. M., \& Roberts, B. 1983, Sol. Phys., 88, 179

Erdélyi, R., \& Verth, G. 2007, A\&A, 462, 743

Gary, G. A. 1996, Sol. Phys., 174, 241

Goossens, M., Andries, J., \& Aschwanden, M. J. 2002, A\&A, 394, L39

Goossens, M., Arregui, I., \& Andries, J. 2006, Phil. Trans. R. Soc. A., 364, 433 Hollweg, J. V., \& Yang, G. 1988, JGR, 93, 5423

Klimchuk, J. A. 2000, Sol. Phys., 193, 53

Klimchuk, J. A., Antichos, S. K., \& Norton, D. 2000, ApJ, 542, 504

Lin, H., Khun, J. R., \& Coulter, R. 2004, ApJ, 613, L177

Lopéz Fuentes, M. C., Klimchuk, J. A., \& Démoulin, P. 2006, ApJ, 639, 459

Lopéz Fuentes, M. C., Démoulin, P., \& Klimchuk, J. A. 2007, ApJ, 673, 586

McClymont, A. N., \& Mikic, Z. 1994, ApJ, 422, 899

McEwan, M. P., Donnelly, G. R., Díaz, A. J., \& Roberts, B. 2006, A\&A, 460, 893

Nakariakov, V. M., Ofman, L., DeLuca, E. E., Roberts, B., \& Davila, J. M. 1999, Sci., 285,862

O’Shea, E., Srivastava, A. K., Doyle, J. G., \& Banerjee, D. 2007, A\&A, 473, L13

Polyanin, A. D., \& Zaitsev, V. F. 2003, Handbook of Exact Solutions for Ordinary Differential Equations (Boca Raton: Chapman \& Hall)

Rae, I. C., \& Roberts, B. 1982, ApJ, 256, 761

Roberts, B., \& Webb, A. R. 1978 Sol. Phys., 56, 5

Ruderman, M. S., \& Roberts, B. 2002, ApJ, 577, 475

Schrijver, C. J. 2001, Sol. Phys., 198, 325

Spruit, H. C. 1981, A\&A, 98, 155

Terra-Homem, M., Erdélyi, R., \& Ballai, I. 2003, Sol. Phys., 217, 199

Van Doorsselaere, T., Andries, J., Poedts, S., \& Goossens, M. 2004, ApJ, 606, 1223

Van Doorsselaere, T., Nakariakov, V. M., \& Verwichte, E. 2007, A\&A, 473, 959

Vernazza, J. E., Avrett, E. H., \& Loeser, R. 1981, ApJS, 45, 635

Verth, G. 2007, Astron. Nachr., 328, 764

Verth, G., Van Doorsselaere, T., Erdélyi, R., \& Goossens, M. 2007, A\&A, 475, 341

Verwichte, E., Nakariakov, V. M., Ofman, L., \& Deluca, E. E. 2004, Sol. Phys., 223, 77

Watko, J. A., \& Klimchuk, J. A. 2000, Sol. Phys., 193, 77

Winebarger, A. R., DeLuca, E. E., \& Golub, L. 2001, ApJ, 553, L81

Winebarger, A. R., Warren, H., van Ballegooijen, A., DeLuca, E. E., \& Golub, L. 2002, ApJ, 567, L89

Winebarger, A. R., Warren, H. P., \& Seaton, D. B. 2003, ApJ, 593, 1164 